\section{Deterministic vs probabilistic strategies in detection*}

MICHAEL KUBOVY, The Hebrew University of Jerusalem, Jerusalem, Israel AMNON RAPOPORT, The University of North Carolina, Chapel Hill, North Carolina 27514 and

AMOS TVERSKY, The Hebrew University of Jerusalem, Jerusalem, Israel

A binary detection task, free from sensory components, is investigated. A deterministic model prescribing a fixed cutoff point is confirmed; a probabilistic model, which generalizes Lee's micromatching model for externally distributed stimuli, is rejected.

The present study is concerned with a decision task in which an individual is required to decide from which of two distributions a particular observation has been drawn. It is assumed that the individual has had adequate exposure to the distributions and that he knows the payoffs for correct and incorrect decisions. The study attempts to discover whether Ss use a deterministic strategy prescribing a fixed cutoff point or a probabilistic strategy to which responses are to be mixed according to the conditional probabilities of the two distributions.

This problem is of central importance to theories of decision making and in particular to signal detection theory (Green \& Swets, 1966), yet it is difficult to investigate within the context of sensory tasks, since the noise and the signal are not specified exactly on a given trial. In such tasks, therefore, departures from optimal performance are attributable to an improper selection of the cutoff point, to the use of a probabilistic strategy, to imperfect sensitivity, or to all of the above.

In a series of studies, Lee (1963), Lee and Janke (1964, 1965), and Lee and Zentall (1966) have attempted to answer this question by using externally distributed stimuli. Three different sensory continua were employed in these studies: position of a dot in a plane, grayness of a patch of paper, and two-digit numbers. In each case, stimuli were drawn from either one of two normal distributions. Using the exact specification of the stimuli, Lee and his associates compared the deterministic response model with a probabilistic response model, named the micromatching hypothesis, derivable from statistical learning theory. In general, the results of the first two studies fell about midway between the two models, whereas the

*This research was supported by a grant to the first two authors from the Faculty of Social Sciences of the Hebrew University of Jerusalem. The costs of preparing the report werc partially supported by Grant M-10006 from the National Institute of Mental Hcalth to the Unversity of North Carolina. The authors wish to thank Mr. E. Zvulun for assistance in data collection. results of the latter two studies corresponded more closely to the micromatching model. Since in these studies Ss were not given prior information about the exact shape of the two distributions, and since the number of trials (either 400 or 500 ) was probably too small to ensure adequate learning, the effects of learning might have obscured the decision strategy employed by Ss. In an attempt to obtain more conclusive evidence on the nature of this strategy, we have (1) freed the decision process from sensory components by using as stimuli numbers drawn from either of two distributions; (2) ensured adequate knowledge of the distributions by providing $S s$ with several thousand learning trials; and (3) motivated Ss to behave optimally by using monetary payoffs contingent upon their performance.

\section{Stimuli}

\section{METHOD}

The stimuli were two sets (denoted by a and b) of 500 four-digit numbers constructed so as to provide the best approximation to two normal distributions with means $m_{a}=1630, m_{b}=1797$, and standard deviations $s_{a}=s_{b}=167\left(d^{\prime}=1\right)$.

\section{Subjects}

The Ss were seven volunteers-three females and four males-all first-year psychology majors at the Hebrew University of Jerusalem. The data of one male $S$ were discarded after it was established that he had seriously misunderstood parts of the instructions.

\section{Table 1} for Each $S$ and Each Payoff Condition

\begin{tabular}{lrrrrrr}
\hline & \multicolumn{7}{c}{ Payoff Condition } \\
\cline { 2 - 6 }$S$ & 1 & 2 & 3 & 4 & 5 & Mean \\
\hline 1 & 1.00 & 0.25 & 0.25 & 3.50 & 2.75 & 1.55 \\
2 & 4.75 & 1.00 & 5.75 & 4.00 & 4.25 & 3.95 \\
3 & 7.00 & 0.75 & 1.00 & 8.00 & 4.25 & 4.20 \\
4 & 3.50 & 5.50 & 18.50 & 6.75 & 1.50 & 7.15 \\
5 & 3.25 & 8.25 & 2.00 & 16.25 & 12.25 & 8.40 \\
6 & 17.25 & 5.75 & 9.75 & 9.00 & 8.00 & 9.95 \\
Mean & 6.13 & 3.58 & 6.21 & 7.92 & 5.50 & 5.87 \\
\hline
\end{tabular}
being sampled on each trial. $S$ was presented on each trial with a single stimulus drawn randomly and with equal probability from either a or b. He was instructed to decide on each trial whether the stimulus was drawn from a or from $b$, and to indicate his decision by writing either " $A$ " or " $B$ " on a response sheet. Feedback was provided at the end of each trial.

The Ss were run in a group. In each session, consisting of 400 trials, an equal number of stimuli from the two distributions were projected on a screen. In the four practice sessions Ss were paid ISf7 (approximately \$2.00) per session, regardless of their performance. Different pay off conditions were used in these sessions, and Ss were asked to compute the number of points they had gained after each block of 100 trials. They were encouraged to try different response strategies in the practice sessions and to maximize the number of points gained. The five experimental sessions were conducted like the practice sessions except that wages were proportional to the number of points gained by each $S$. The five payoff conditions used in these sessions-one in each session-are presented in Fig. 1. The mean earnings of the group was fixed at approximately $\mathbf{I S f 7 . 5 0}$ per session. Ss were paid half their earnings at the end of each session; the balance was paid upon completion of the experiment.

\section{RESULTS}

To compare the deterministic and probabilistic models, critical points were

\section{Percentage of Trials on Which the Cutoff Point Model is Violated} Payoff Condition
Procedure

There were 4 practice sessions and 27 experimental sessions in which different procedures were employed. Each session lasted about $2 \mathrm{~h}$. This paper deals with the results of one procedure that was employed during 5 experimental sessions: Nos. 5, 8, 12, 17, and 21 .

The Ss were told that the distributions, a and $b$, represented heights of women and men, respectively, that they need not represent any adult population they were familiar with, that they were unimodal, symmetric, and partially overlapping, and that men and women had equal chances of 


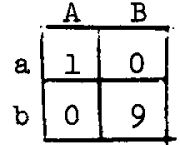

(1)

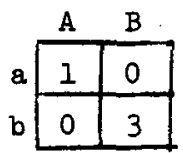

(2)

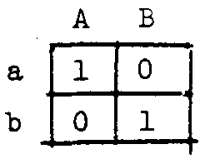

(3)

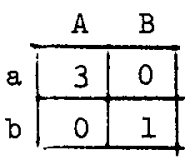

(4)

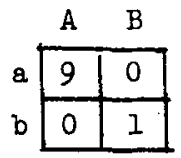

(5)
Fig. 1. Payoff matrices for the five payoff conditions, A, B denote responses; $a, b$ denote distributions.

computed for each $S$ in each payoff condition. A critical point is a stimulus value for which the number of violations of a deterministic model is minimal. Formally, let $A(x)$ be the total number of trials on which $S$ responded $A$ to stimuli that were larger than $x$, and let $B(x)$ be the total number of trials on which $S$ responded $B$ to stimuli that were smaller than $x$. A critical point is a stimulus-value $x$ for which $A(x)+B(x)$ is minimal. When a critical point was not unique, the median critical point was selected for further analysis. To test the deterministic model, we treated the critical points as hypothetical cutoff points, thereby obtaining an index of departure from the model. The percentages of trials on which a deterministic model was violated for each $\mathrm{S}$ and each payoff condition are presented in Table 1. The proportions of $B$ responses as a function of the stimulus value (the psychometric function) for a typical $S$ (S 3) in all five payoff conditions are displayed in Fig. 2.

The presence of violations in Table 1 reflects a certain degree of mixing, showing that the deterministic model is not perfectly satisfied. Nevertheless, the percentage of violations is not substantial, and an adequate statistical procedure is required to test and compare the two models.

The micromatching model (Lee, 1963) asserts that for any stimulus, $x$,

$$
\frac{P(B \mid x)}{P(A \mid x)}=\frac{P(b \mid x)}{P(a \mid x)}
$$

This model, however, does not take payoffs into account. We have, therefore, generalized the model by assuming that

$$
\frac{P(B \mid x)}{P(A \mid x)}=\frac{E[U(B, x)]}{E[U(A, x)]}
$$

$$
=\frac{P(b \mid x) U(B, b)+P(a \mid x) U(B, a)}{P(b \mid x) U(A, b)+P(a \mid x) U(A, a)}
$$

where, e.g., $E[U(B, x)]$ is the expected utility of choosing $B$ after observing $x$, and $U(B, b)$ is the utility of choosing $B$ when the observation was drawn from $b$. Since in the present study $U(A, b)=U(B, a)$, we can let $U(A, b)=U(B, a)=0, U(A, a)=\alpha$, and
$\mathrm{U}(\mathrm{B}, \mathrm{b})=\beta$, whence

$$
\frac{P(B \mid x)}{P(A \mid x)}=\frac{P(b \mid x) \beta}{P(a \mid x) \alpha}
$$

(Note that the generalized micromatching model, denoted GM, is also applicable to cases where the prior probabilities are unequal.) If $A(c)=B(c)$ for any critical point, $c$, it follows that $\mathrm{P}(\mathrm{A} \mid \mathrm{c})=\mathrm{P}(\mathrm{B} \mid \mathrm{c})=$ .5 , and hence, by the above equation,

$$
\frac{\mathrm{P}(\mathrm{b} \mid \mathrm{c})}{\mathrm{P}(\mathrm{a} \mid \mathrm{c})}=\frac{\alpha}{\beta}
$$

A chi-square test of the hypothesis that $A(c)=B(c)$ over all payoff conditions was nonsignificant $(p>10)$.

To evaluate the adequacy of the two models, the frequency of correct responses expected under each of them was compared to the observed frequency of correct responses. For the deterministic model, the expected frequency was computed by treating the critical points as hypothetical cutoff points and applying them to the actual stimuli presented to the Ss. For the GM model, Eq. 2 was employed to obtain the value of $\alpha / \beta$ for each $S$ and

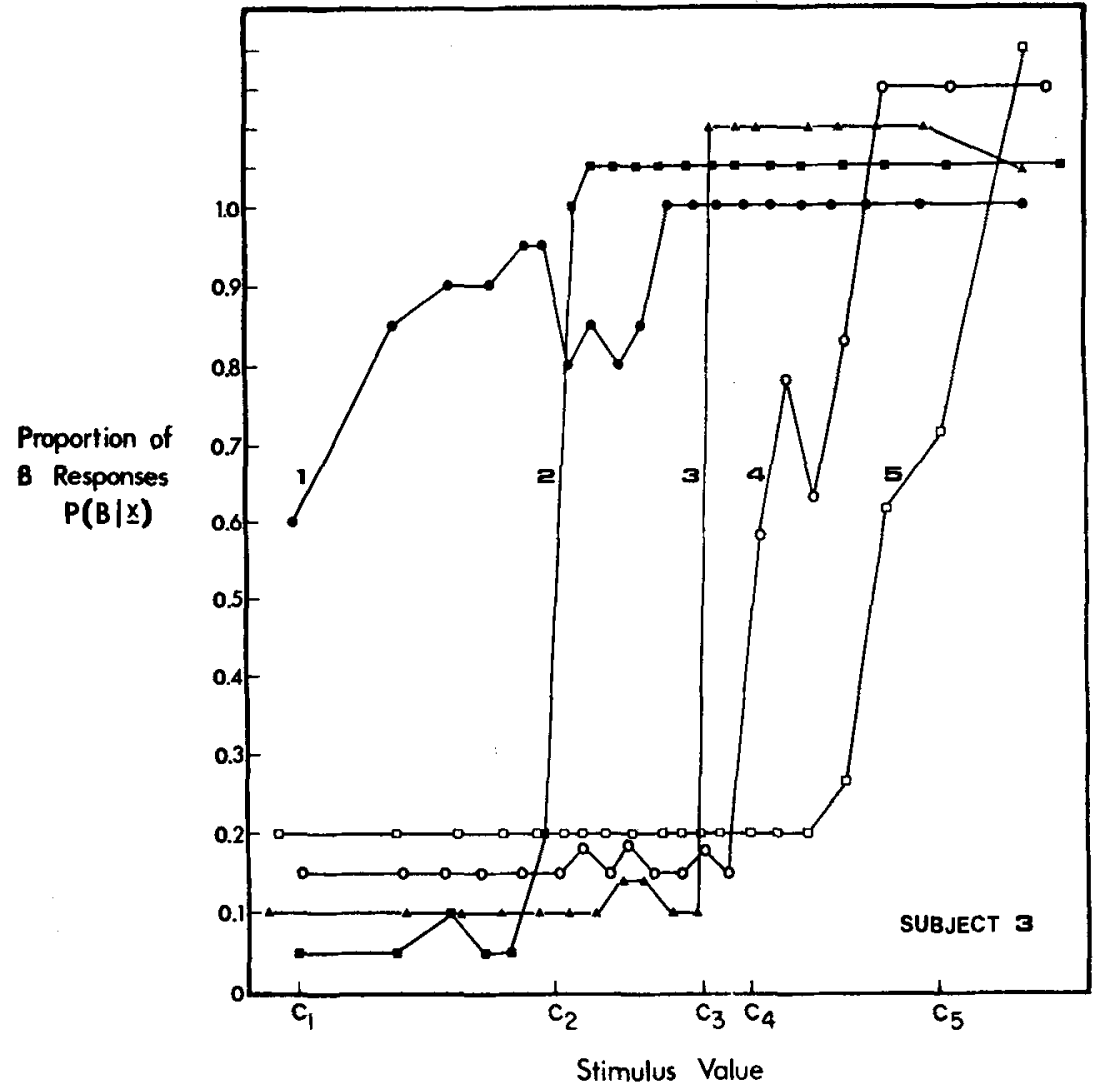

Fig. 2. Proportion of $B$ responses in blocks of 20 as a function of mean stiniulus value (on a $\log$ likelihood-ratio scale, i.e., $\log [P(x \mid b) / P(x \mid a)]$ ) for each payoff condition for S 3. Successive curves are displaced upward by 0.05 . Respective critical points $\left(c_{1}\right.$, $\ldots \ldots, c_{5}$ ) are marked along the abscissa. 
Table 2

Chi-Square Values for the Comparison of Observed Frequency of Correct Responses With Frequencies Expected Under the Two Response Models for Each S Across Payoff Conditions

\begin{tabular}{lcc}
\hline & $\begin{array}{c}\text { Cutoff } \\
\text { Point } \\
\text { Model }\end{array}$ & $\begin{array}{c}\text { Micro- } \\
\text { matching } \\
\text { Model }\end{array}$ \\
\hline 1 & 0.33 & $22.89^{(i i i)}$ \\
2 & 2.49 & $40.32^{(i i i)}$ \\
3 & 5.13 & $13.06^{(i)}$ \\
4 & 2.40 & $15.82^{(i i)}$ \\
5 & 3.52 & $26.78^{(i i i)}$ \\
6 & 6.81 & $43.06^{(i i)}$ \\
Overall & 20.68 & $159.90^{(i i i)}$ \\
\hline
\end{tabular}

(i) $p<.05,(i i)_{p}<.01$, (iii) $p<.001$. Note-Each individual comparison is based on $6 \mathrm{df}$; the overall comparison is based on $30 \mathrm{df}$.

Table 3

Chi-Square Values for the Comparison of Observed Frequency of Correct Responses With Frequencies Expected Under the Two Response Models for Each Payoff Condition Across Ss

\begin{tabular}{ccc}
$\begin{array}{c}\text { Payoff } \\
\text { Condition }\end{array}$ & $\begin{array}{c}\text { Cutoff } \\
\text { Point } \\
\text { Model }\end{array}$ & $\begin{array}{c}\text { Micro- } \\
\text { matching } \\
\text { Model }\end{array}$ \\
\hline 1 & 8.71 & 2.17 \\
2 & 3.36 & $39.83^{(i i i)}$ \\
3 & 1.86 & $63.22^{(i i i)}$ \\
4 & 4.52 & $45.03^{(i i)}$ \\
5 & 2.23 & 2.65 \\
Overall & 20.68 & $159.90^{(i i i)}$ \\
\hline
\end{tabular}

(iii) $p<.001$

Note-Each individual comparison is based on $5 \mathrm{df}$; the overall comparison is based on $30 \mathrm{df}$.

payoff condition separately. These values were substituted into Eq. 1 to obtain the expected frequency of $\mathrm{A}$ and $\mathrm{B}$ responses from which the expected frequencies of correct responses for each stimulus were calculated. The overall expected frequency for a given $S$ on a given session was obtained by adding the expected frequencies of correct responses over all stimuli presented in this session. The chi-square valucs for the comparisons between the observed and expected frequencies of correct responses under the two models are presented in Table 2 for each $\mathrm{S}$ and in Table 3 for each payoff condition.

The results show that the discrepancy between the observed and expected frequencies is nonsignificant under the deterministic model and highly significant under the GM model for each of the $S$ s over all payoff conditions. Table 3 shows that the same result holds for the three nonextreme payoff conditions (2,3, and 4) over all Ss. In the extreme payoff conditions ( 1 and 5), the expected proportion of correct responses predicted by both models approaches .5. Hence the statistical power of the test of the GM

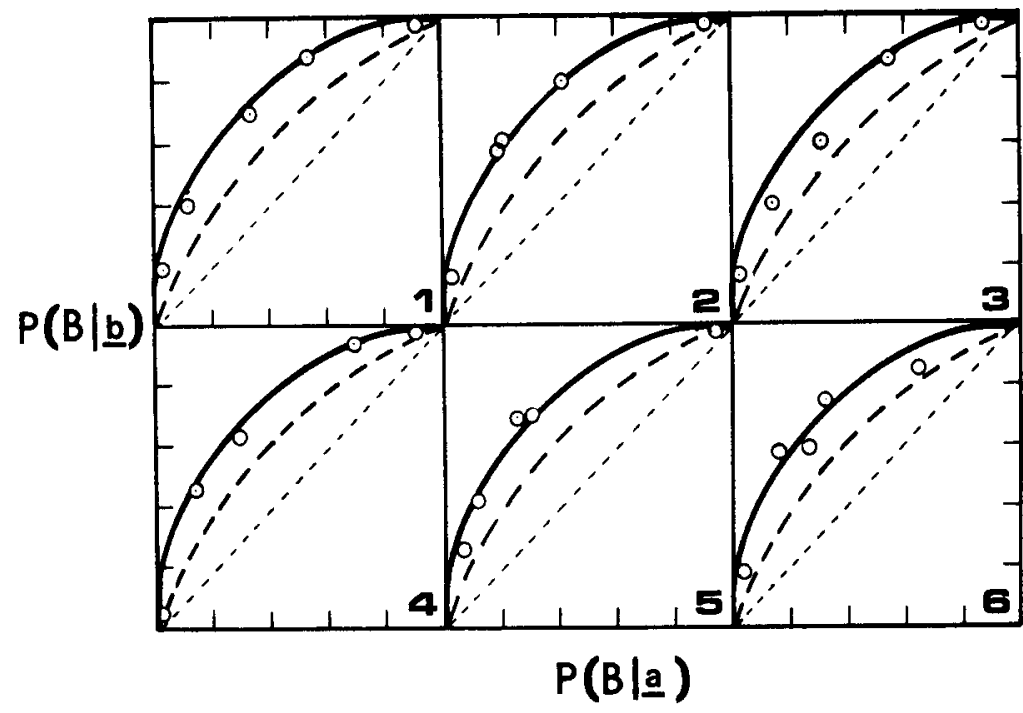

Fig. 3. ROC plots of the data with the curves predicted by the cutoff point model (solid line) and the micromatching model (dashed line) for all Ss.

Table 4

Parameter Estimates of the ROC Curve Predicted by the Cutoff Point Model

\begin{tabular}{lllllll}
\hline & $S 1$ & $S 2$ & $S 3$ & $S 4$ & $S 5$ & $S 6$ \\
\hline Intercept & 1.0206 & .9693 & $.8335(i)$ & .9186 & 1.0170 & .8700 \\
Slope & 1.0145 & .9767 & .9965 & 1.0252 & 1.0248 & .9175 \\
\hline
\end{tabular}

$(i)_{p}<.05$

model (assuming the validity of the deterministic model) is considerably lower in these latter conditions than in Conditions 2, 3, and 4. The overall discrepancy (across all Ss and payoff conditions) is highly significant for the GM model and nonsignificant for the deterministic model.

Figure 3 displays the ROC plots of the data and the theoretical curves predicted by the two models for each of the Ss. The intercept and slope of the linear regression line (empioying double probability coordinates) fitted to the data of each $S$ in the five payoff conditions by the maximum likelihood method (Dorfman \& Alf, 1968) according to the deterministic model, are presented in Table 4 . The $95 \%$ confidence intervals for 11 out of the 12 estimates include 1.0, which is the value predicted by the signal detection theory, assuming normal distributions with equal variances and $d^{\prime}=1$.

In summary, although some degree of mixing is observed, the GM model is clearly inadequate, contrary to the conclusion of Lee and his colleagues. The difference between the findings may be due to a difference in procedure. Whereas the Ss of the present study received extensive practice with the two distributions $(2,000$ trials before the first experimental session, and several thousand more thereafter), Lee's Ss learned the distributions in the course of a relatively short experiment (400 or 500 trials). Under these circumstances, the locations of the cutoff points probably shifted during the study. It is not surprising, therefore, that Lee's Ss produced data that are closer to the probabilistic model than the present data. Strictly speaking, the deterministic model is also violated, for it is intolerant of any error. Nevertheless, it yields a very good account of Ss' behavior when evaluated in terms of the frequency of correct responses. And this, after all, is the basis on which both distributions and strategies are learned.

\section{REFERENCES}

DORFMAN, D. D. \& ALF, E., JR. Maximum likelihood estimation of parameters of signal detection theory-a direct solution. Psychometrika, 1968, 33, 117-124.

GREEN, D. M., \& SWETS, J. A. Signal detection theory and psychophysics. New York: Wiley, 1966.

LEF, W. Choosing among confusably distributed stimuli with specified likelihood ratios. Perceptual \& Motor Skills, 1963, 16, 445-467.

LEE, W., \& JANKE, M. Categorizing externally distributed stimulus samples from three continua. Journal of Experimental Psy chology, $1964,68,376-382$.

LEE, W., \& JANKE, M. Categorizing externally distributed stimulus samples from unequal molar probabilities. Psychological Reports, $1965,17,79-90$.

LEE, W. \& ZENTALL. T. R. Factorial effects in the categorization of externally distributed stimulus samples. Perception \& Psychophysics. $1966,1,120-124$.

(Accepted for publication August 31, 1970.) 